\title{
Acellular Dermal Matrix to Treat Full Thickness Skin Defects: Follow-Up Subjective and Objective Skin Quality Assessments
}

\author{
Jae Yeon Park', \\ Tae Geun Lee', \\ Ji Ye Kim², \\ Myung Chul Lee ${ }^{1}$, \\ Yoon Kyu Chung ${ }^{2}$, \\ Won Jai Lee ${ }^{1}$ \\ ${ }^{1}$ Department of Plastic and Reconstructive \\ Surgery, Institute for Human Tissue \\ Restoration, Yonsei University College of \\ Medicine, Seoul; ${ }^{2}$ Department of Plastic and \\ Reconstructive Surgery, Yonsei University \\ Wonju College of Medicine, Wonju, Korea
}

No potential conflict of interest relevant to this article was reported.

\begin{abstract}
Background: There are several options for replacement of the dermal layer in fullthickness skin defects. In this study, we present the surgical outcomes of reconstruction using acellular dermal substitutes by means of objective and subjective scar assessment tools.

Methods: We retrospectively reviewed the medical records of 78 patients who had undergone autologous split-thickness skin graft with or without concomitant acellular dermal matrix (CGDerm or AlloDerm) graft. We examined graft survival rate and evaluated postoperative functional skin values. Individual comparisons were performed between the area of skin graft and the surrounding normal skin. Nine months after surgery, we compared the skin qualities of CGDerm graft group $(n=25)$, AlloDerm graft group $(n=8)$ with skin graft only group $(n=23)$ each other using the objective and subjective measurements.

Results: The average of graft survival rate was $93 \%$ for CGDerm group, $92 \%$ for AlloDerm group and $86 \%$ for skin graft only group. Comparing CGDerm grafted skin to the surrounding normal skin, mean elasticity, hydration, and skin barrier values were $87 \%$, $86 \%$, and $82 \%$, respectively. AlloDerm grafted skin values were $84 \%, 85 \%$, and $84 \%$, respectively. There were no statistical differences between the CGDerm and AlloDerm groups with regard to graft survival rate and skin functional analysis values. However, both groups showed more improvement of skin quality than skin graft only group.

Conclusion: The new dermal substitute (CGDerm) demonstrated comparable results with regard to elasticity, humidification, and skin barrier effect when compared with conventional dermal substitute (AlloDerm).
\end{abstract}

Keywords: Acellular dermis / Skin graft / Quality evaluation

\section{INTRODUCTION}

When the full-thickness skin defect occurs, full-thickness skin graft (FTSG) or local flap is used in usual reconstruction method. However, for coverage of the large sized defect, the dermis cannot be reconstructed with these procedures, but it can be reconstructed with split-thickness skin graft (STSG). Therefore autologous

\section{Correspondence: Won Jai Lee}

Department of Plastic and Reconstructive Surgery, Severance Hospital, Yonsei University College of Medicine, 50 Yonsei-ro, Seodaemun-gu, Seoul 120-749,

Korea

E-mail: pswjlee@yuhs.ac

Received December 25, 2013 / Revised February 26, 2014 / Accepted March 6, 2014 split-thickness graft is still considered as the mainstay for the treatment of the large skin defects caused by traumatic wound and after release of scar contracture [1]. Despite of these versatility and advantages of skin graft, problems such as graft loss, scar contracture, loss of elasticity, sensory impairment, adhesions of flexor tendons, and unfavorable aesthetic results including hypo- or hyper-pigmentation were regarded as the drawbacks accompanied with STSG [2-5]. To overcome these limitations, various and alternative methods have been attempted including regional flaps, FTSG, use of cultured human epithelium, alloplastic dermal substitutes or acellular dermal matrix (ADM) [6-9]. 
Among these diverse methods, artificial dermal substitute or ADM graft combined with simultaneous STSG have been carried out effectively and easily nowadays. There are various kinds of dermal substances that have been developed, such as Integra (bovine collagen-based), Matriderm (native bovine type I collagen fiber template), and AlloDerm (freeze-dried extracellular matrix [ECM] derived from cadaver skin). Dermal substitute is an appropriate way to minimize scar contracture and to improve the quality of the grafted area in strained regions requiring elasticity, pliability, and stability [9]. AlloDerm (LifeCell Corp., Branchburg, NJ, USA) is the most widely used. It is a freeze-dried product obtained from the cadaveric skin and allowed to improve the structure of the dermis to contribute to wound healing and reduces scar contracture [10-12]. Matriderm (Dr. Suwelack Skin \& Health care AG, Billerbeck, Germany) is a highly porous collagen-elastin matrix consisting of a native bovine type I collagen fiber template coated with an a-elastin hydrolysate derived from bovine ligamentum nuchae [9]. The structurally intact native collagen serves as an essential component of new ECM for the migration of cells and vascularization, and therefore MatriDerm provides a scaffold to restore the skin and modulates the formation of scar. Because this product contains elastin and collagen, reduces the tension and increases the elasticity of the newly formed skin when the wound is restored.

We used two types of dermal substitutes (CGDerm and AlloDerm) for full-thickness skin defects. One of the most popular dermal substitutes, AlloDerm, was extensively studied in vitro and animal tests and was found to be superior to other collagen materials with regard to wound-healing parameters [13-15]. A clinical study also proved that grafting AlloDerm improved dermal architecture and reduced scar contracture for small, acute uncomplicated wounds that did not require skin grafting for wound closure [16]. CGDerm is an another ADM derived from donated human skin, like AlloDerm, and provides a complex, three dimensional array of proteins that interact with one another and with host cells.

In this study, we investigated the clinical outcome of graft success rate and evaluated skin quality by means of objective and subjective scar assessment tools for two types of ADM (CGDerm and AlloDerm) grafted skin. Also, we analyzed the quality of the grafted skin by comparing the value of elasticity, transdermal water loss, erythema and melanin of both adjacent normal skin and grafted areas.

\section{METHODS}

\section{Patients and method}

We retrospectively analyzed the medical records of 78 patients with full-thickness skin defects who underwent surgery using acellular dermal substitute, such as CGDerm or AlloDerm, and skin grafting between December 2009 and June 2011. The Institutional Review Board approved this retrospective study. Fifty-five patients in this study had a comparable sheet of ADM placed over the defect, and operators took care to place the basement membrane side to the wound surface. The dermal thickness was not greater than 0.020-inches. Next, a split thickness skin (0.00800.010 inches) was harvested from the lateral thigh and placed over the artificial dermis.

The 30 patients had full-thickness skin defects covered with ADM (AlloDerm). The other 25 patients underwent coverage with a newly developed ADM (CGDerm) and STSG. Both groups were retrospectively studied for comparative defects. Twentythree patients had skin graft without dermal substitute. Among 78 patients, cases with partial graft loss were also included.

All patients underwent a standardized functional skin evaluation 15 months after surgery.

A clinical trial was initiated to study the efficacy and survival rate in cases of full-thickness skin defects which required split thickness skin grafting to obtain wound closure. We also compared skin qualities between the dermal substitute with the STSG and the surrounding normal skin. The objective parameters for skin quality were applied approximately 15 months after surgery considering scar maturation period. The Cutometer, Corneometer, Tewameter, and Mexameter were used to evaluate objective skin quality. Additionally, we used a modified Vancouver scar scale (VSS) as a subjective scar assessment tool. 


\section{Materials}

We chose two acellular dermal matrices, AlloDerm and CGDerm, for this study.

Alloderm regenerative tissue matrix (LifeCell Co., The Woodlands, TX, USA) is a freeze-dried ECM tissue derived from cadaver skin through a process resulting in the removal of all cellular components and residues. AlloDerm is the most popular artificial dermis that is used for skin defect. Acellular dermal matrix should be tolerable to graft rejection, and the role of the ECM is to prevent non-specific inflammation and degradation.

CGDerm (CGBio Co., Dae-woong Pharm., Seoul, Korea) is an $\mathrm{ADM}$ that is derived from cadaveric skin, from which the epidermis and dermal cells have been removed by freeze-drying. This matrix provides a complex, 3-dimensional array of proteins that interact with one other and with host cells. These proteins include networks of collagen, elastin, hyaluronan, and proteoglycan like
AlloDerm. In comparative molecular analysis, more angiogenin, endostatin, pentraxin 3 and platelet factor 4 were observed in CGDerm. Because vascular channels and dermal architecture are retained in CGDerm, it has the additional possibility of revascularization, remodeling, and incorporation into the host tissue.

\section{Evaluation tools}

The Cutometer (Courage-Khazaka Electronic GmbH, Cologne, Germany), which is a reliable and valid instrument that creates reproducible data, was used for the evaluation of skin elasticity. Each measurement was performed at the center of the area of interest (Fig. 1A). The Corneometer was used as a tool for analyzing the process of scar humidification over time, and the scar surface areas were measured (Fig. 1B). The Tewameter was used to measure transepidermal water loss, with the measurement of water evaporation based on diffusion (Fig. 1C). The Mexameter is a tool used
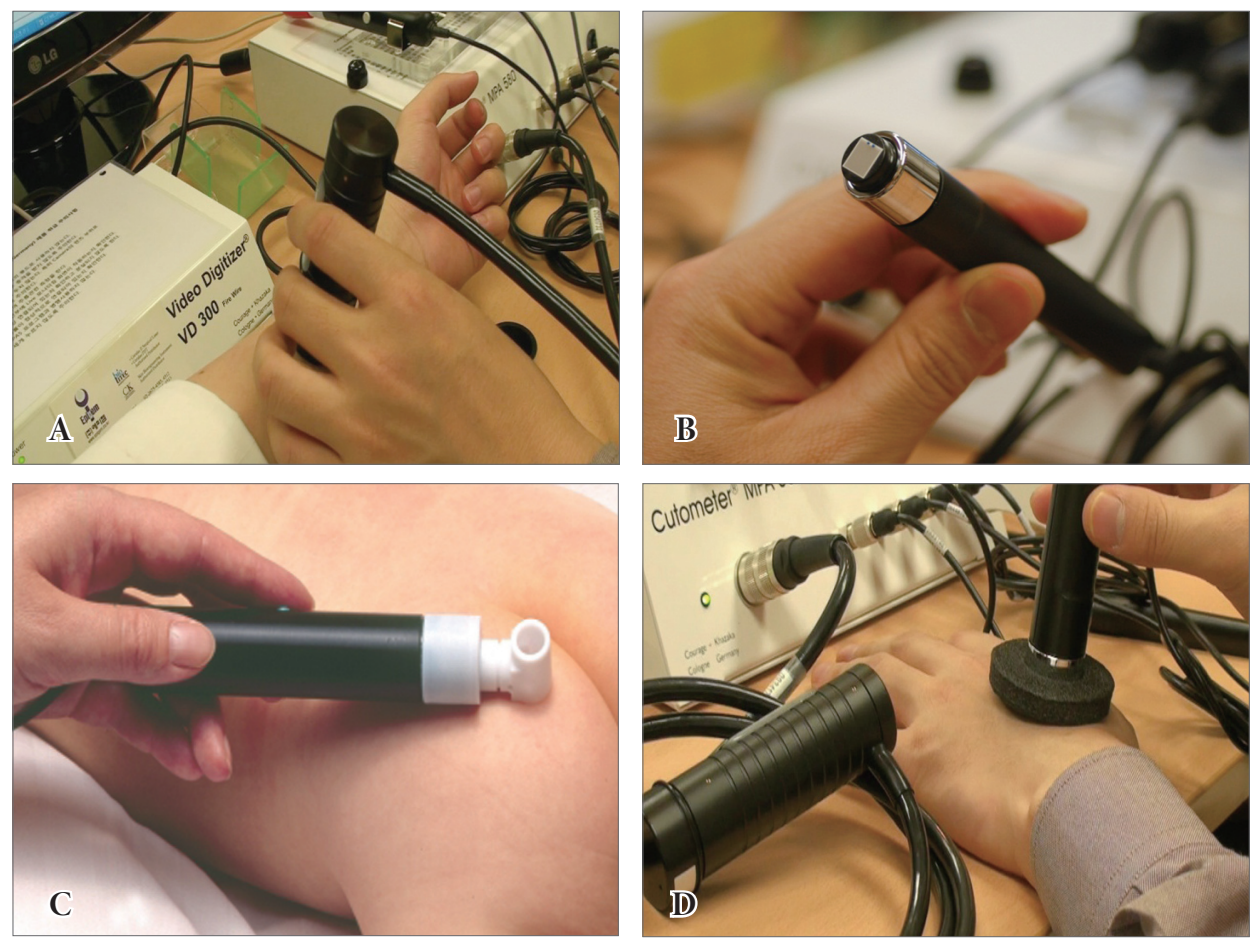

Fig. 1. Skin quality evaluation tools. (A) The Cutometer, which is a reliable and valid instrument that creates reproducible data, was used for the evaluation of skin elasticity. Each measurement was performed in the center of the area of interest. (B) The Corneometer was used as a tool for analyzing the process of scar humidification over time, and the scar surface areas were measured. (C) The Tewameter was used to measure transepidermal water loss, with the measurement of water evaporation based on diffusion. (D) The Mexameter is a tool used to measure melanin (pigmentation) and redness (erythema). Measurement of the melanin and erythema values is based on a source of light with three specific wavelengths whose radiation is absorbed and diffusely reflected by the skin. 
to measure melanin (pigmentation) and redness (erythema) (Fig. 1D). Measurement of the melanin and erythema values is based on a source of light with three specific wavelengths whose radiation is absorbed and diffusely reflected by the skin. A photo detector was used to analyze the diffuse reflection from the skin. If the skin is well supplied with blood, then the hemoglobin value is increased. It is possible to evaluate the stimulation of the microcirculation before and after intervention by measuring the hemoglobin value.

The postoperative scar was subjectively assessed using the modified VSS, which is generally used to assess burn scarsand involves clinical evaluation of four parameters of scar tissue: vascularization, pliability, height, and pigmentation [17]. Pigmentation and vascularization are visually determined.

A trained independent research nurse who was to the operative methods and procedures evaluated all scars using the VSS during outpatient visits 15 months after surgery.

\section{Statistical analysis}

T-test was used to compare the mean skin values and graft survival rates of the AlloDerm group to the CGDerm group. Comparison between the ADM group, which represents the mean of AlloDerm and CGDerm group, and the skin graft only group was also performed using t-test. Statistical significance was evaluated with a $95 \%$ confidence interval.

\section{RESULTS}

All patients underwent one-stage ADM and split-thickness skin grafting. Wounds were then dressed with a negative pressure dressing (vacuum assisted closure). All the graft donor site wounds were healed on the 7th to 8th postoperative day (average, 7.7 days). Wound related complications were not noted.

The numbers and types of injuries of grafted skins were listed in Tables 1,2. The CGDerm group had a 93\% graft survival rate. Among the 25 patients treated with CGDerm, four patients had partial skin loss. However, these patients experienced adequate secondary healing. The AlloDerm group had a $92 \%$ graft survival rate. Similar to the CGDerm group, four patients experienced partial graft loss and all healed well with secondary wound healing. There were no significant statistical differences in skin graft survival rate between the conventional dermal acellular matrix (AlloDerm) and the newly developed dermal substitute (CGDerm) groups (Table 3). Comparing the CGDerm grafted skin area to the surrounding normal skin, the mean elasticity value was $87 \%$. The hydration and skin barrier values were $86 \%$ and $82 \%$, respectively (Table 3). The AlloDerm grafted skin values for elasticity, hydration, and skin barrier were $84 \%, 85 \%$ and $84 \%$, respectively, compared to those of the surrounding normal skin. There were no statistical differences between the CGDerm and AlloDerm groups in graft survival rate or objective skin analysis values (elasticity, hydration, and skin barrier) (Table 3). Skin darkness as measured with the Mexameter was different from the adjacent normal skin in the CGDerm and Alloderm groups, and the intergroup difference was not statistically significant (Table 3). Through the result, about elasticity value of the grafted skin with-

Table 1. Patient demographics, location of operative wound (number)

\begin{tabular}{lccc|}
\hline Neck & $\begin{array}{c}\text { CGDerm graft } \\
\text { group }\end{array}$ & $\begin{array}{c}\text { AlloDerm graft } \\
\text { group }\end{array}$ & $\begin{array}{c}\text { Skin graft only } \\
\text { group }\end{array}$ \\
\hline Chest wall & 1 & 0 & 2 \\
\hline Back & 2 & 1 & 1 \\
\hline Inguinal area & 0 & 1 & 0 \\
\hline Forearm & 0 & 2 & 1 \\
\hline Thigh & 12 & 16 & 4 \\
\hline Lower leg & 4 & 1 & 5 \\
\hline Foot & 1 & 3 & 4 \\
\hline Total & 5 & 6 & 23 \\
\hline
\end{tabular}

Table 2. Patient demographics, type of injuries (number)

\begin{tabular}{lccc}
\hline & $\begin{array}{c}\text { CGDerm } \\
\text { graft group }\end{array}$ & $\begin{array}{c}\text { AlloDerm } \\
\text { graft group }\end{array}$ & $\begin{array}{c}\text { Skin graft } \\
\text { only group }\end{array}$ \\
\hline Trauma & 7 & 14 & 8 \\
\hline Burn & 12 & 13 & 9 \\
\hline Nevus & 1 & 1 & 0 \\
\hline Postoperative scar & 1 & 2 & 4 \\
\hline Etc. (DM foot, Marjolin's ulcer) & 4 & 1 & 2 \\
\hline Total & 25 & 30 & 23 \\
\hline
\end{tabular}

DM, diabetes mellitus. 
out dermal substitute was 0.24 as normal surrounding skin value was 0.38 which accounts for $63 \%$. Extension, retraction and pliability values were $0.32,0.27$, and 0.30 in non-dermal substitute group. They were about 78\%, 69\%, and 73\% as compared with normal surrounding skin (Table 3). Comparable results between ADM group and the skin graft only group were listed in table III. In the Cutometer which represents elasticity of skin, ADM grafted skin showed $0.51 \pm 0.12$. Non dermal matrix grafted skin showed $0.42 \pm 0.17$. The $p$-value between two groups revealed less than 0.001. Also Corneometer which represents hydration of skin showed that grafted skin with ADM was statistically better hydration value (acellular dermal matrix grafted skin: $41 \pm 8.02$; non dermal matrix grafted skin: $34 \pm 7.48$; $p$-value $<0.001$ ). Tewameter which represents skin barrier showed higher value in ADM grafted skin. But there was no statistical difference (Table 4).

The subjective scar assessment results revealed similar VSS values in the CGDerm group and the AlloDerm group. The overall scar index was 3.33 in the CGDerm group and 3.5 in the AlloDerm group. The acellular dermal substitute group had a better VSS score than the non-dermal substitute group (Table 5).

Table 3. Comparable mean skin values and graft survival rates in the acellular dermal substitute and non- dermal substitute groups 15 months after surgery

\begin{tabular}{cccccc} 
Evaluation tool & $\begin{array}{c}\text { Cutometer } \\
\text { (elasticity) }\end{array}$ & $\begin{array}{c}\text { Corneometer } \\
\text { (hydration) }\end{array}$ & $\begin{array}{c}\text { Tewameter } \\
\text { (skin barier) }\end{array}$ & $\begin{array}{c}\text { Mexameter } \\
\text { (melanin/ } \\
\text { enthema } \\
\text { value) }\end{array}$ & $\begin{array}{c}\text { Graft } \\
\text { survivial } \\
\text { rate }\end{array}$ \\
\hline $\begin{array}{c}\text { CGDerm group } \\
(\%)\end{array}$ & 87 & 86 & 82 & 1.31 & 93 \\
$\begin{array}{c}\text { AlloDermgroup } \\
(\%)\end{array}$ & 84 & 85 & 84 & 1.51 & 92 \\
$\begin{array}{c}\text { Skin graft only } \\
\text { group (\%) }\end{array}$ & 63 & 78 & 69 & 2.12 & 86 \\
\hline
\end{tabular}

Table 4. Comparable results between skin graft with acellular dermal matrix and skin graft without dermal matrix

\begin{tabular}{lcccc} 
Evaluation tool & $\begin{array}{c}\text { Cutometer } \\
\text { (elasticity) }\end{array}$ & $\begin{array}{c}\text { Corneometer } \\
\text { (hydration) }\end{array}$ & $\begin{array}{c}\text { Tewameter } \\
\text { (skin barier) }\end{array}$ & $\begin{array}{c}\text { Graft survival } \\
(\%)\end{array}$ \\
\hline $\begin{array}{c}\text { Acellular dermal } \\
\text { matrix group }(n=55)\end{array}$ & $0.51 \pm 0.12$ & $41 \pm 8.02$ & $10.4 \pm 5.21$ & 92 \\
$\begin{array}{c}\text { Skin graft only group } \\
(n=23)\end{array}$ & $0.42 \pm 0.17$ & $34 \pm 7.48$ & $9.8 \pm 6.82$ & 86 \\
\hline -value & $<0.001$ & $<0.001$ & 0.32 & \\
\hline
\end{tabular}

Table 5. Comparative Vancouver scar scale values in the CGDerm and AlloDerm groups 15 months after surgery

\begin{tabular}{lccccc} 
Skin quality & \multicolumn{5}{c}{ Pigmentation Height Flexibility Vascularization Sum } \\
CGDerm group & 1.08 & 1.20 & 1.08 & 0.25 & 3.33 \\
AlloDerm group & 1.25 & 1.00 & 0.87 & 0.38 & 3.5 \\
Skin graft only group & 2.91 & 2.74 & 2.15 & 0.80 & 5.8 \\
\hline
\end{tabular}

\section{Case reports}

\section{Case 1}

A 53-year-old man had a hypopharyngeal cancer. Wide excision and right radical neck dissection was performed by otorhinolaryngology team. The patient had a $5 \times 6 \mathrm{~cm}$ full thickness skin defect on left forearm area after flap elevation (Fig. 2A). The CGDerm was applied to the defect area immediately (Fig. 2B). The patient showed $100 \%$ of graft survival rate. After 18 months, the surface of the CGDerm grafted area appeared cosmetically satisfactory, and there was not impairment during excursion of forearm flexors (Fig. 2C, D). On the skin analysis, the mean percentage of the value for grafted skin to surrounding normal skin about elasticity, hydration and skin barrier values were $89 \%, 85 \%$, and 87\% respectively. Overall modified VSS noted 3.0 (Fig. 2C, D).

\section{Case 2}

An 8-year-old girl had a hypertrophic scar on her right dorsum of the foot. The hypertrophic scar was completely excised out (Fig. 3A). Immediately after excision of the hypertrophic scar, CGDerm and autogenous skin graft was done at a $6 \times 4 \mathrm{~cm}$ sized defect (Fig. 3B, C). Twenty-four months later, reliable result was obtained cosmetically and functionally (Fig. 3D). This patient showed 100\% at graft survival rate. On the skin analysis, the mean percentage of the value for grafted skin to surrounding normal skin about elasticity, hydration and skin barrier values were $86 \%, 88 \%$, and $85 \%$ respectively. Overall modified VSS noted 3.5 (Fig. 3D).

\section{DISCUSSION}

In this study, we evaluated the ADM allograft group in comparison with the skin graft only group with regard to skin graft survival rate and skin quality. Many experiments have demonstrated 

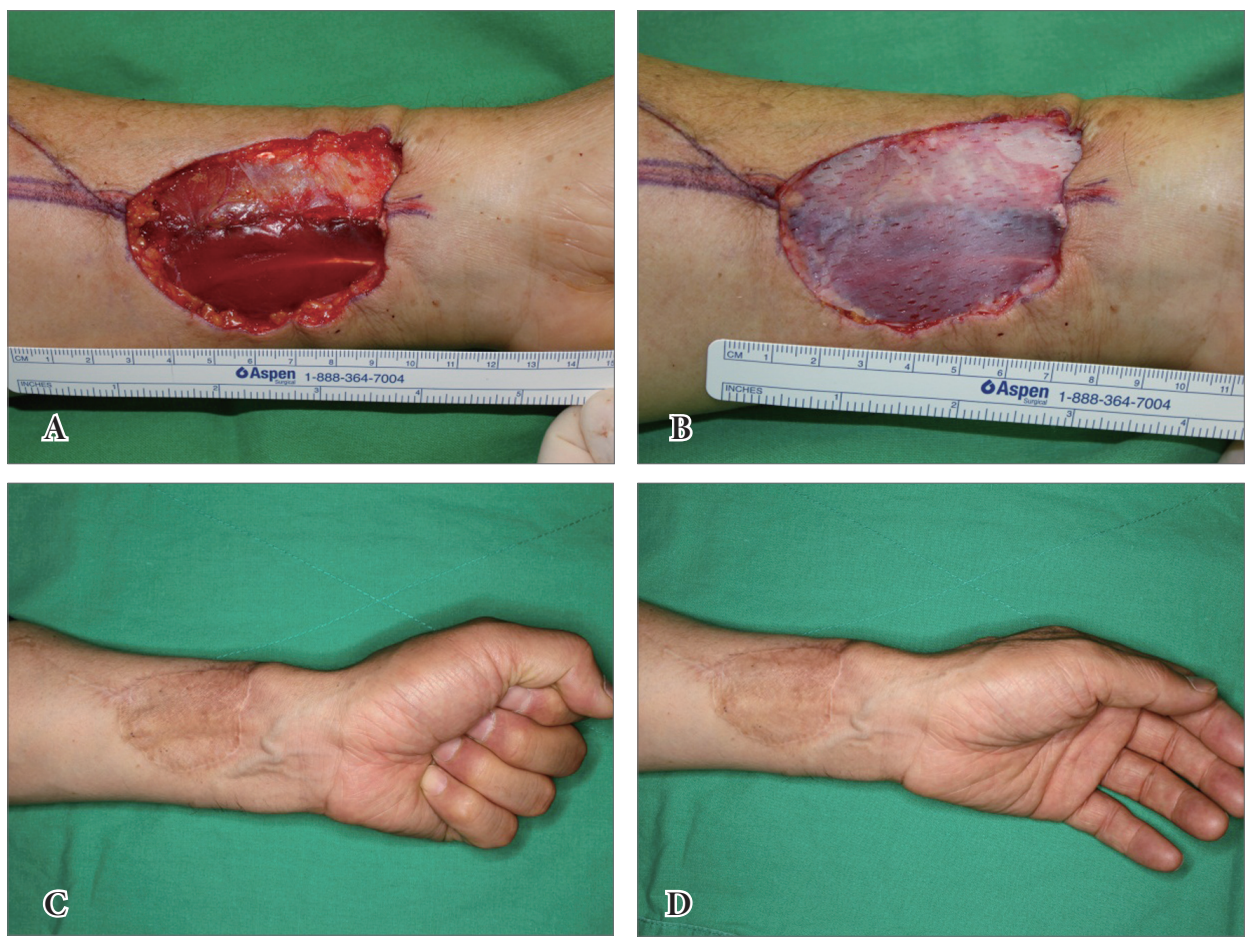

Fig. 2. Clinical application of a new dermal substitute to the radial forearm free flap donor site. (A) A 53 -year-old man had a $5 \times 6 \mathrm{~cm}$ raw surface on the left radial forearm after flap elevation. (B) CGDerm was immediately applied to the defect area. (C, D) After 18 months, the surface of the CGDerm grafted area was cosmetically satisfactory, and there was not impairment during excursion of forearm flexors.
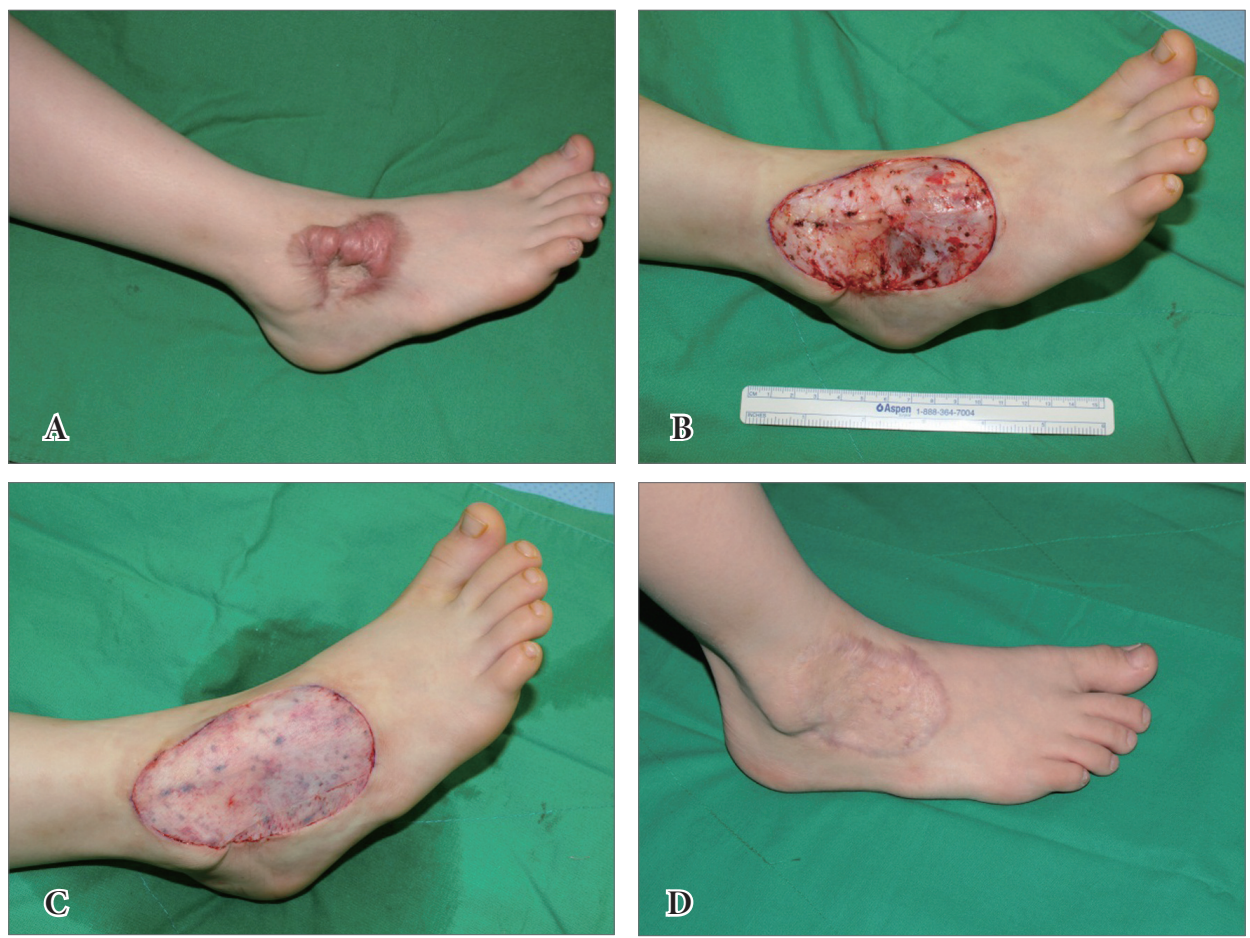

Fig. 3. (A) An 8-year-old girl had a hypertrophic scar on the dorsum of her right foot. (B, C) Immediately after excision of the hypertrophic scar, CGDerm graft and an autologous skin graft were undergone on the defect site. (D) Twenty-four months later, positive cosmetic and functional results were reported. 
the effectiveness of dermal substitutes. Since its introduction in the late 1980s, Integra dermal regeneration template, an irreversibly cross-linked collagen/glycosaminoglycan matrix, has been widely used for dermal replacement in reconstructive surgery [10$12,18]$. Because it has some drawbacks, alternative matrices have been developed that consist of less densely packed collagen fibers. Collagen-elastin matrix (MatriDerm, Dr. Suwelack, Skin and Health Care AG, Billerbeck, Germany) is not chemically crosslinked and is one of the alternatives for reconstructive surgery that has demonstrated positive effects on the acceleration of improvement in the quality and functionality of skin reconstruction $[19,20]$.

Another alternative option is acellular dermal matrix. A prior study has compared the effects of dermal substitutes on wound healing, demonstrating that human skin-derived products, such as ADM, produced the minimal amount of contraction and a thicker newly developed dermis in the healed wounds in comparison with control or synthetic dermal substitutes [19]. Prior research has shown that the application of acellular cadaver dermis is extremely useful in the fields of burn care and reconstructive surgery $[18,20]$.

Acellular dermal matrix contains collagen and elastin, which control tensile strength and elasticity; proteoglycans that induce angiogenesis; laminin that maintains binding with the connective tissues; and basement membrane that consists of collagen type IV. CGDerm shares a similar molecular structure with AlloDerm and is also produced via freeze-drying.

In our results, we applied both subjective and objective scar tools to evaluate the quality of the grafted skin. The elasticity, humidification, skin barrier, and darkness of the postoperative scar were objectively assessed after 15 months using the Cutometer, Corneometer, Tewameter, and Mexameter. We found no significant differences between the two matrices in regard to graft success rate, postoperative scar elasticity, humidification, or skin barrier. Additionally, the subjective scar assessment revealed similar VSS values in the CGDerm group and the AlloDerm group. Also the ADM group was more effective than skin graft only group in skin quality when comparing both objective and subjective skin parameters. However, there are limitations in our research. First, there can be some bias due to selecting proper surrounding normal skin. It was complicated and doctor's own subjective comparison was performed. Even though same anatomical portion would be included, some wounds like burn involving whole lower leg including knee area had difficulties about choosing proper normal skin at surrounding area. The second limitation of this study was relatively short follow-up period which was 15 months after the operation. More long-term follow-up is needed for scar maturation.

Our results suggest that the skin grafting accompanied with ADM allograft gives positive effects about skin quality for skin reconstructive surgery. Moreover, a new dermal substitute (CGDerm) demonstrated comparable results with regard to elasticity, humidification, and skin barrier effect when compared to a conventional dermal substitute(AlloDerm).

\section{REFERENCES}

1. Muller MJ, Nicolai M, Wiggins R, MacGill K, Herndon DN. Modern treatment of a burn wound. In: Herndon DN, editor. Total burn care. London: W.B. Saunders; 1996. p. 136-47.

2. Unal S, Ersoz G, Demirkan F, Arslan E, Tutuncu N, Sari A. Analysis of skin-graft loss due to infection: infection-related graft loss. Ann Plast Surg 2005;55:102-6.

3. Kroonen L, Shumaker PR, Kwan JM, Uebelhoer N, Hofmeister E. Treatment of split-thickness skin graft-related forearm scar contractures with a carbon dioxide laser protocol: 3 case reports. J Hand Surg Am 2013;38:2164-8.

4. Jeon H, Kim J, Yeo H, Jeong H, Son D, Han K. Treatment of diabetic foot ulcer using matriderm in comparison with a skin graft. Arch Plast Surg 2013;40:403-8.

5. Lutz BS, Wei FC, Chang SC, Yang KH, Chen IH. Donor site morbidity after suprafascial elevation of the radial forearm flap: a prospective study in 95 consecutive cases. Plast Reconstr Surg 1999;103:132-7.

6. Kim RJ, Izzard ME, Patel RS. Supraclavicular artery island flap for reconstructing defects in the head and neck region. Curr Opin Otolaryngol Head Neck Surg 2011;19:248-50.

7. Scherer-Pietramaggiori SS, Pietramaggiori G, Orgill DP. Skin graft. In: Neligan PC, editor. Plastic surgery. 3rd ed. London: Elsevier Saunders; 2013.p. 319-38.

8. Matsumura H, Gondo M, Imai R, Shibata D, Watanabe K. Chronological histological findings of cultured epidermal autograft over bilayer artificial dermis. Burns 2013;39:705-13.

9. Haslik W, Kamolz LP, Manna F, Hladik M, Rath T, Frey M. Management of full-thickness skin defects in the hand and wrist region: first long-term experiences with the dermal matrix Matriderm. J Plast Re- 
constr Aesthet Surg 2010;63:360-4.

10. Moiemen NS, Staiano JJ, Ojeh NO, Thway Y, Frame JD. Reconstructive surgery with a dermal regeneration template: clinical and histologic study. Plast Reconstr Surg 2001;108:93-103.

11. Moiemen NS, Vlachou E, Staiano JJ, Thawy Y, Frame JD. Reconstructive surgery with Integra dermal regeneration template: histologic study, clinical evaluation, and current practice. Plast Reconstr Surg 2006;117:160S-74S.

12. Nguyen DQ, Dickson WA. A review of the use of a dermal skin substitute in burns care. J Wound Care 2006;15:373-6.

13. Middelkoop E, de Vries HJ, Ruuls L, Everts V, Wildevuur CH, Westerhof W. Adherence, proliferation and collagen turnover by human fibroblasts seeded into different types of collagen sponges. Cell Tissue Res 1995;280:447-53.

14. de Vries HJ, Middelkoop E, Mekkes JR, Dutrieux RP, Wildevuur CH, Westerhof H. Dermal regeneration in native non-cross-linked collagen sponges with different extracellular matrix molecules. Wound Repair Regen 1994;2:37-47.

15. Lamme EN, de Vries HJ, van Veen H, Gabbiani G, Westerhof W, Middelkoop E. Extracellular matrix characterization during healing of full-thickness wounds treated with a collagen/elastin dermal substitute shows improved skin regeneration in pigs. J Histochem $\mathrm{Cy}$ tochem 1996;44:1311-22.

16. De Vries HJ, Zeegelaar JE, Middelkoop E, Gijsbers G, Van Marle J, Wildevuur $\mathrm{CH}$, Westerhof W. Reduced wound contraction and scar formation in punch biopsy wounds. Native collagen dermal substitutes. A clinical study. Br J Dermatol 1995;132:690-7.

17. Yim H, Cho YS, Seo CH, Lee BC, Ko JH, Kim D, Hur J, Chun W, Kim JH. The use of AlloDerm on major burn patients: AlloDerm prevents post-burn joint contracture. Burns 2010;36:322-8.

18. Jones I, Currie L, Martin R. A guide to biological skin substitutes. Br J Plast Surg 2002;55:185-93.

19. van Zuijlen PP, van Trier AJ, Vloemans JF, Groenevelt F, Kreis RW, Middelkoop E. Graft survival and effectiveness of dermal substitution in burns and reconstructive surgery in a one-stage grafting model. Plast Reconstr Surg 2000;106:615-23.

20. Ryssel H, Gazyakan E, Germann G, Ohlbauer M. The use of MatriDerm in early excision and simultaneous autologous skin grafting in burns: a pilot study. Burns 2008;34:93-7. 